Copyright @ 2005 IEEE.

Reprinted from the Proceedings of the Annual Meeting of the IEEE Lasers and Electro-Optics Society (18th : 2005 : Sydney, Australia)

pp. 818-819

This material is posted here with permission of the IEEE. Such permission of the IEEE does not in any way imply IEEE endorsement of any of the University of Adelaide's products or services. Internal or personal use of this material is permitted. However, permission to reprint/republish this material for advertising or promotional purposes or for creating new collective works for resale or redistribution must be obtained from the IEEE by writing to pubs-permissions@ieee.org.

By choosing to view this document, you agree to all provisions of the copyright laws protecting it. 


\title{
Integrated all-optical chalcogenide waveguide pulse regenerator: experiment and modeling
}

\author{
V.G. Ta'eed, M. Shokooh-Saremi, L.B. Fu, D.J. Moss, M. Rochette, I.C.M. Littler, and B.J. Eggleton \\ Centre for Ultrahigh-bandwidth Devices for Optical Systems (CUDOS) \\ School of Physics, University of Sydney, Sydney, NSW 2006, Australia \\ Y. Ruan and B. Luther-Davies \\ Centre for Ultrahigh-bandwidth Devices for Optical Systems (CUDOS) \\ Laser Physics Centre, the Australian National University, Canberra, ACT 0200, Australia
}

\begin{abstract}
We present an integrated, all-optical, chalcogenide waveguide pulse regenerator based on linear filtering of self phase modulated pulses. We demonstrate a nonlinear transfer function with 1.5 ps optical pulses and find good agreement with theory.
\end{abstract}

All-optical regenerators are critical for ultrahigh bit-rate lightwave transmission systems at $40 \mathrm{~Gb} / \mathrm{s}$ and beyond as a means of preventing the accumulation of signal distortion [1]. In recent years, significant attention has been focused on Chalcogenide glasses [2-3] as promising nonlinear optical materials for integrated alloptical devices due to their large $\mathrm{n}_{2}$ (up to $1000 \times$ silica) and low two-photon absorption (and hence good figure of merit). This is combined with the ability to tailor material properties via stoichiometry, as well as photosensitivity allowing the fabrication of photo-written gratings and waveguides.

Using integrated Bragg grating filtering, we present an all-optical signal regenerator based on pulse carving of self-phase modulation (SPM) broadened spectra, as conceived by Mamyshev [4]. The device clearly exhibits a nonlinear power transfer function and pulse shaping properties which can be used to regenerate pulses and improve optical signal to noise ratio (OSNR) and Bit Error Rate [5]. We find good agreement between theory and experimentally obtained output signal spectra, pulse autocorrelation and power transfer curve.

The device, shown schematically in Fig. 1, consists of a $4.0 \times 2.4 \mu \mathrm{m}^{2}$ chalcogenide $\left(\mathrm{As}_{2} \mathrm{~S}_{3}\right)$ rib waveguide fabricated by pulsed laser deposition, lithography and dry etching [6], followed by an integrated band-pass filter (Fig. 2a), photo-written as two high quality Bragg gratings using a Sagnac interferometer with a CW $532 \mathrm{~nm}$ doubled Nd:YAG laser. The short coherence length of the writing laser provided compensated refractive index apodization, and the inferred a.c. index change for the two strong gratings was $\Delta n>0.01$.

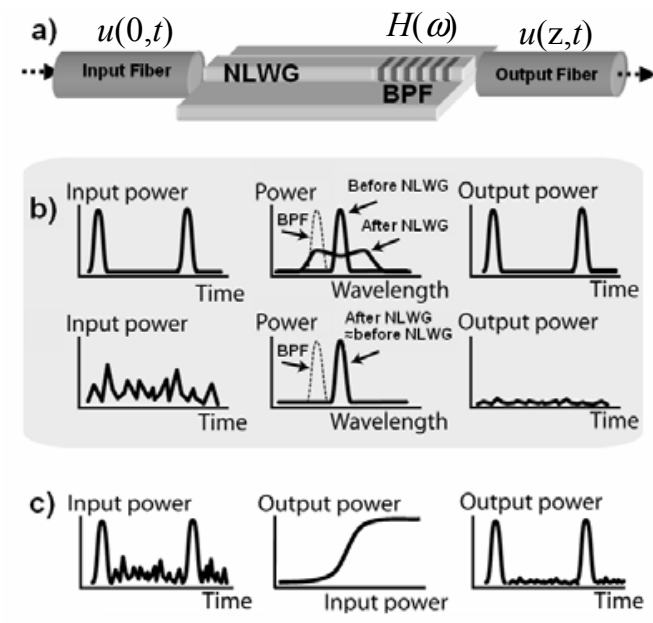

Fig. 1 a) Physical device architecture. b) Noise experiences less SPM spectral broadening in the nonlinear waveguide (NLWG) than signal, and hence is attenuated more after the off-centre band pass filter (BPF). c) This results in nonlinear power transfer curve which can suppress noise.

Device performance was demonstrated with an amplified, modelocked "Figure of 8 " laser producing near transform-limited $1.5 \mathrm{ps}$ pulses, butt-coupled into the waveguide. Fig. 2b) shows the pulse spectra after passing through a bare waveguide with no grating present. This shows significant broadening with increasing input peak power, due to SPM, with a maximum nonlinear optical phase shift $\left(\phi_{\mathrm{NL}}\right)$ of approximately $3 \pi / 2$. The laser wavelength was then tuned to within the rejection band of the Bragg grating. This resulted in low transmission at low input power since the filter rejection bandwidth is much wider than the $3 \mathrm{~dB}$ spectral bandwidth of the input pulse. As the input pulse power was increased, SPM broadened the spectrum so that power was 


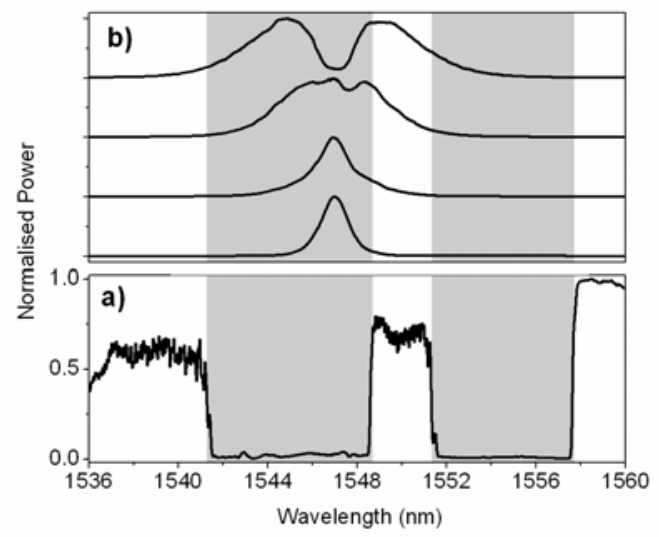

Fig. 2 a) Transmission spectra for the band-pass filter. b) Spectral broadening of 2.0 ps pulses with increasing power in a waveguide with no grating.

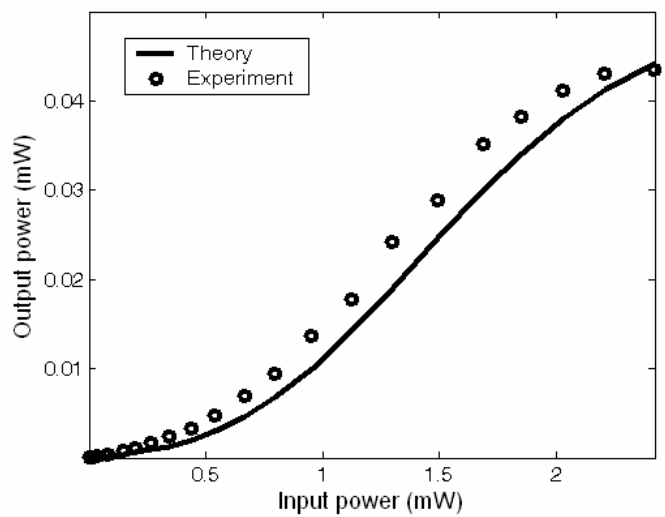

Fig. 3 Resulting "S" shaped power transfer curve enabling suppression of noise.

transmitted through the pass band of the filter. Fig. 3 shows the resulting power transfer curve which exhibits a clear nonlinear " $\mathrm{S}$ " shape, required for optical regeneration.

Fig. 4 shows the autocorrelation of the input and output pulses at high power and indicates that the output pulses were broadened slightly to $\sim 3$ ps. While $\mathrm{As}_{2} \mathrm{~S}_{3}$ has large normal material dispersion $(-410 \mathrm{ps} / \mathrm{nm} / \mathrm{km})$ [7], the dispersion length $\left(\mathrm{L}_{\mathrm{d}}\right)$ is almost two orders of magnitude longer than the device length. Consequently the broadening is attributed to grating-edge dispersion and spectral-mismatch between the filter and pulse. Optimizing the grating pass-band shape and width is expected to reduce this and provides a degree of freedom for reconfigurable regeneration [8].

Since dispersion in the waveguide is negligible, we model the device as lumped SPM followed by filtering:

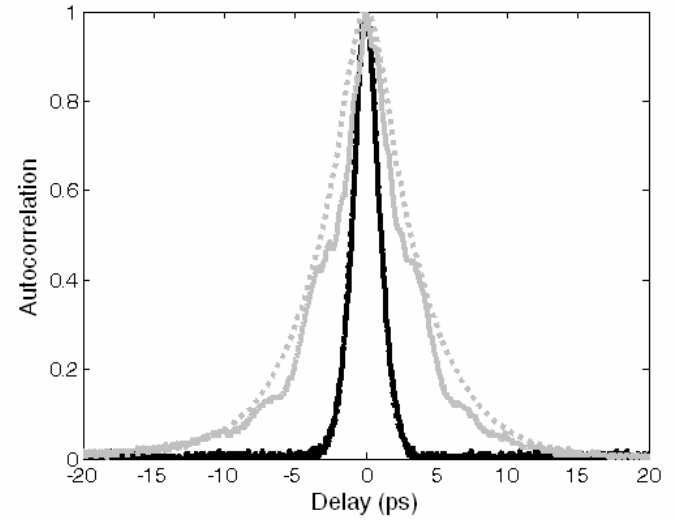

Fig. 4 Experimental (solid) and theoretical (dashed) input (black) / output (gray) pulse autocorrelation. Pulses broaden from $1.5 \mathrm{ps}$ to $\sim 3 \mathrm{ps}$, due to a combination of grating edge dispersion and pulse/filter mismatch.

$$
U(z, \omega)=F\left[u(0, t) \exp \left[i \phi_{N L}(t)\right]\right] H(\omega)
$$

where $u$ is the normalized amplitude, $\phi_{N L}$ is the nonlinear induced phase shift and $H(\omega)$ is the filter function. The filter was modeled using the thin-film matrix method [9], producing complex Fresnel transmission coefficients that fully describe the dispersive properties of the filter. Good agreement between this model and the measured device pulse autocorrelation and pulse transfer curve is observed in fig. 3 and fig. 4.

In conclusion, we demonstrate experimentally the first integrated pulse regenerator using a nonlinear $\mathrm{As}_{2} \mathrm{~S}_{3}$ waveguide followed by linear filtering through a double Bragg grating band-pass filter. We achieve good agreement between theory and experimental results in terms of power transfer and output pulse width.

We acknowledge support from the Australian Research Council (ARC). V.G.T. is grateful for support from CSIRO. M.S.-S. appreciates support from the Iranian Ministry of Science, Research and Technology and Iran Telecommunication Research Centre.

\section{References}

[1] O. Leclerc et al, J. of Lightwave Technol., 212779 (2003).

[2] A. Zakery and S.R. Elliott, J. Non-Crystal. Solids, 3301 (2003).

[3] R. E. Slusher et al, J. Opt. Soc. Am. B, 211146 (2004).

[4] P. V. Mamyshev, Proc. ECOC, 475 (1998).

[5] M. Rochette et al, IEEE Photon. Technol. Lett., 17908 (2005).

[6] Y. Ruan et al, Optics Exp., 125140 (2004).

[7] M. Asobe et al, J. Appl. Phys. 775518 (1995).

[8] I. C. M. Littler et al, Opt. Exp., 133397 (2005).

[9] P. Yeh, Optical waves in layered media, Wiley, N.Y., (1988). 